H. MASAOKA AND S. SEGAWA

KODAI MATH. J.

17 (1994), 351-359

\title{
HARMONIC DIMENSION OF COVERING SURFACES
}

\author{
Dedicated to Professor Mitsuru Nakai on his sixtieth birthday
}

By Hiroaki Masaoka and Shigeo Segawa

\section{Introduction}

Let $R$ be an open Riemann surfaces of null boundary which has a single ideal boundary component in the sense of Kerékjártó-Stoïlow. A relatively noncompact subregion $\Omega$ of $R$ is said to be an end of $R$ if the relative boundary $\partial \Omega$ consists of finitely many analytic Jordan curves (cf. Heins [4]). We denote by $\mathscr{Q}(\Omega)$ the class of nonnegative harmonic functions on $\Omega$ with vanishing boundary values on $\partial \Omega$. The harmonic dimension of $\Omega, \operatorname{dim} \mathscr{Q}(\Omega)$ in notation, is defined as the minimum number of elements of $\mathscr{P}(\Omega)$ generating $\mathscr{L}(\Omega)$ provided that such a finite set exists, otherwise as $\infty$. It is known that $\operatorname{dim} \mathscr{P}(\Omega)$ does not depend on a choice of end of $R: \operatorname{dim} \mathscr{Q}(\Omega)=\operatorname{dim} \mathscr{Q}\left(\Omega^{\prime}\right)$ for any pair $\left(\Omega, \Omega^{\prime}\right)$ of ends of $R$ (cf. [4]). In terms of the Martin compactification $\operatorname{dim} \mathscr{Q}(\Omega)$ coincides with the number of minimal points over the ideal boundary (cf. Constantinescu and Cornea [3]).

In this paper we especially concern with ends $W$ which are subregion of $p$-sheeted unlimited covering surfaces of $\{0<|z| \leqq \infty\}$. For these $W$ it is known that $1 \leqq \operatorname{dim} \mathscr{Q}(W) \leqq p$ (cf. [4]). Consider two positive sequences $\left\{a_{n}\right\}$ and $\left\{b_{n}\right\}$ satisfying $b_{n+1}<a_{n}<b_{n}<1$ and $\lim _{n \rightarrow \infty} a_{n}=0$. Set $G=\{0<|z|<1\}-I$ where $I=$ $\cup_{n=1}^{\infty} I_{n}$ and $I_{n}=\left[a_{n}, b_{n}\right]$. We take $p(>1)$ copies $G_{1}, \cdots, G_{p}$ of $G$. Joining the upper edge of $I_{n}$ on $G$, and the lower edge of $I_{n}$ on $G_{3+1}(j \bmod p)$ for every $n$, we obtain a $p$-sheeted covering surface $W=W_{p}^{I}$ of $\{0<|z|<1\}$ which is naturally considered as an end of a $p$-sheeted covering surface of $\{0<|z| \leqq \infty\}$. In [4] Heins proved the followings:

(A) If I is sufficiently 'thin' at $z=0$ such as

$$
\lim _{R \ni x \rightarrow-0} \hat{R}_{G_{0}}^{I}(x)<+\infty,
$$

then $\operatorname{dim} \mathscr{Q}(W)=p$, where $\hat{R}_{G_{0}}^{I}$ is the balayage of $G_{0}(z)=\log (1 /|z|)$ relative to $I$ on $D$;

(B) if $I$ is sufficiently 'thick' at $z=0$ such as

Received September 6, 1993. 


$$
\sum_{n=1}^{\infty} \log \frac{b_{n}}{a_{n}}=\infty
$$

then $\operatorname{dim} \mathscr{Q}(W)=1$.

The purpose of this paper is to extend these Heins' results. For example our Theorem 1 (cf. $\S 1$ ) in more general setting for $I$ implies that if $I$ is thin at $z=0$, in the sense that $z=0$ is an irregular boundary point of $G$ with respect to Dirichlet problem, then $\operatorname{dim} \mathscr{P}(W)=p$, which sharpens the above (A). Restricted to the case $p=2^{m}(m \in N)$ our Theorem 2 (cf. $\S 1$ ) in a bit more general setting for $I$ implies that if $I$ is not thin at $z=0$, then $\operatorname{dim} \mathscr{P}(W)=1$, which partially sharpens the above (B). Consequently we have the following which completely determines the harmonic dimension of $W=W_{p}^{I}$ in the case $p=2^{m}$ (cf. [6]) :

THEOREM. Suppose that $p=2^{m}$. Then

(i) $\operatorname{dim} \mathscr{Q}(W)=p$ if and only if $I$ is thin at $z=0$;

(ii) $\operatorname{dim} \mathscr{P}(W)=1$ if and anly if $I$ is not thin at $z=0$.

In $\S 1$ we give preliminaries and state main results Theorems 1 and 2 . The proof of Theorem 1 (resp. Theorem 2) is given in $\S 2$ (resp. $\S 3$ ).

\section{$\S 1$. Preliminaries from potential theory and statement of main results}

1.1. We begin with recalling the definition of balayage. Consider an open Riemann surface $F$ possessing the Green's function. Denote by $\mathcal{S}$ the class of nonnegative superharmonic functions on $F$. Let $E$ be a subset of $F$ and $s$ belong to $\mathcal{S}$. Then the balayage $\hat{R}_{s}^{E}={ }^{F} \hat{R}_{s}^{E}$ of $s$ relative to $E$ on $F$ is defined by

$$
\hat{R}_{s}^{E}(z)=\liminf _{x \rightarrow z} \inf \{u(x): u \in \mathcal{S}, u \geqq s \text { on } E\}
$$

(cf. e.g. [1]). Let $G_{\xi}^{F}(\cdot)$ be the Green's function on $F$ with pole at $\xi$. We here review fundamental properties of balayage (cf. [1], [2], [5], etc.).

PROPOSITION 1.1. (i) If $E_{1} \subset E_{2}$, then $\hat{R}_{s}^{E_{1}} \leqq \hat{R}_{s}^{E_{2}}$;

(ii) $\hat{R}_{s}^{E_{1} \cup E_{2}} \leqq \hat{R}_{s}^{E_{1}}+\hat{R}_{s}^{E_{2}}$;

(iii) $\hat{R}_{u+v}^{E}=\hat{R}_{u}^{E}+\hat{R}_{v}^{E}$;

(iv) if $N$ is a polar set, then $\hat{R}_{s}^{E \cup N}=\hat{R}_{s}^{E}$;

(v) if $E$ is a closed subset of $F$, then $\hat{R}_{s}^{E}(z)=s(z)$ on $E$ except possibly for those $z \in \partial E$ which are irregular boundary points of $F-E$;

(vi) $\hat{R}_{G F}^{E}(x)=\hat{R}_{G}^{E} F(z)$ for every $z$ and $x$ in $F$.

Next we state the definition of thinness (cf. [2]).

Definition 1.1. Let $z$ be a point of $F$ and $E$ a subset of $F$. We say that $E$ is thin at $z$ if $\hat{R}_{G F}^{E} \neq G_{z}^{F}$. 
Assuming that $E$ is closed and $z$ belongs to $E$ in the above definition, it is well-known that $E$ is thin at $z$ if and only if $z$ is an irregular boundary point of $F-E$ with respect to Dirichlet problem (cf. e.g. [1, p. 348]).

1.2. In the complex plane $\boldsymbol{C}$, we introduce the weakest topology which makes all positive superharmonic functions in $C$ continuous. This topology is called fine topology (cf. e.g. [2]). It is well-known that a subset $U$ of $\boldsymbol{C}$ is a fine neighborhood of a point $z$ in $\boldsymbol{C}$ if and only if $\boldsymbol{C}-U$ is thin at $z$. Here and hereafter, for simplicity, we denote by $G_{\xi}(\cdot)$ the Green's function on $\{|z|<1\}$ with pole at $\xi$. In $\S 2$ we will be in need of the following proposition (cf. [2]):

Proposition 1.2. Let $E$ be a domain in $\boldsymbol{C}$ such that the point $z=0$ belongs to $\partial E$. Suppose that $\boldsymbol{C}-E$ is thin at $z=0$ and $h$ is a positive superharmonic function on $E$. Then $h / G_{0}$ has a fine limit $f-\lim _{E \ni z \rightarrow 0} h(z) / G_{0}(z)$ at $z=0$, where the fine limit of $h / G_{0}$ at $z=0$ is the limit of $h / G_{0}$ at $z=0$ with respect to the fine topology.

1.3. In order to state main results, we begin with fixing the notations. Denote by $D$ the open unit disc $\{|z|<1\}$. Let $\left\{J_{n}\right\}_{n=1}^{\infty}$ be a family of closed segments $J_{n}$ in $D-\{0\}$ such that $J_{m} \cap J_{n}=\emptyset$ for every $m$ and $n$ with $m \neq n$ and $\left\{J_{n}\right\}_{n=1}^{\infty}$ accumulates only at $z=0$ in $D \cup \partial D$. Set $J=\cup_{n=1}^{\infty} J_{n}$ and $S=D$ $\{0\}-J$. By definition of $S, S$ has two edges on each $J_{n}$. Then we denote by $J_{n}^{+}$one of them and by $J_{n}^{-}$the other. Take $p(>1)$ copies $S_{1}, \cdots, S_{p}$ of $S$ and identify along each $J_{n}$, the edge $J_{n}^{+}$on $S_{\rho}$ being joined to the edge $J_{n}^{-}$on $S_{j+1}(j \bmod p)$. We thereby obtain a $p$-sheeted covering surface $W=W_{p}$ of $\{0<|z|<1\}$ which is naturally considered as an end a $p$-sheeted covering surface of $\{0<|z| \leqq \infty\}$. The followings are our main results.

THEOREM 1. If $J$ is thin at the origin, then $\operatorname{dim} \mathscr{Q}(W)=p$.

Theorem 2. Suppose that $p=2^{m}(m \in N)$ and that $J$ is symmetric with respect to the real axis. If neither of $J \cap \boldsymbol{R}$ and $\boldsymbol{R}-J$ is thin at the origin, then $\operatorname{dim} \mathscr{P}(W)=1$.

It is easily checked that Theorem in Introduction follows from Theorems 1 and 2 .

\section{$\S 2$. Proof of Theorem 1}

2.1. First we give the following proposition :

Proposition 2.1. Suppose that $J$ and $S$ are the same as in Theorem 1. Then, 


$$
f-\lim _{S \ni z \rightarrow 0} \frac{\hat{R}_{G_{0}}^{J}(z)}{G_{0}(z)}=0
$$

if and only if $J$ is thin at $z=0$, where $\hat{R}_{G_{0}}^{J}={ }^{D} \hat{R}_{G_{0}}^{J}$.

Proof. The 'only if' part of the assertion follows from the definition of thinness. Suppose that $J$ is thin at $z=0$. Set $D_{0}=\{z \in D: \operatorname{Re} z \geqq 0\}$ and $D_{1}=$ $\{z \in D: \operatorname{Re} z \leqq 0\}$. By (ii) of Proposition 1.1, we only have to prove that

$$
f-\lim _{S \ni z \rightarrow 0} \frac{\hat{R}_{G_{0}}^{J \cap D_{k}}(z)}{G_{0}(z)}=0
$$

for $k=0$ and 1 . We prove (1) only for $k=0$, since the proof works similarly for $k=1$. By the fact that the open segment $(-1,0)$ is not thin at $z=0$ and by Proposition 1.2 , we have only to prove that

$$
\lim _{R \ni z \rightarrow-0} \frac{\hat{R}_{G_{0}}^{J \cap D_{0}}(z)}{G_{0}(z)}=0 .
$$

We take points $x$ in $D_{0} \cap J$ and $z$ in $(-1,0)$. From simple calculation we obtain the inequality

$$
G_{z}(x)=\log \left|\frac{1-z x}{x-z}\right| \leqq \log \frac{1}{|z|}=G_{z}(0) .
$$

Hence we have

$$
\hat{R}_{G_{z}}^{E} \leqq \hat{R}_{G_{z}(0)}^{E}=G_{z}(0) \hat{R}_{1}^{E}
$$

on $D$ for $z \in(-1,0)$ and a subset $E$ of $J \cap D_{0}$. Let $\rho$ be a real number with $\rho>1$ and set $D(N)=\left\{|z|<e^{-\rho^{N}}\right\}(N \in N)$. Then Wiener's criterion implies that

$$
\lim _{N \rightarrow \infty} \hat{R}_{1}^{J \cap D_{0} \cap D(N)}(0)=0
$$

(cf. [2, p. 80]). By (ii) and (vi) of Proposition 1.1 and by (3), we have

$$
\begin{aligned}
\limsup _{R \ni z \rightarrow-0} \frac{\hat{R}_{G_{0}}^{J \cap D_{0}}(z)}{G_{0}(z)} & \leqq \limsup _{R \ni z \rightarrow-0}\left(\frac{\hat{R}_{G_{0}}^{J \cap D_{0} \cap D(N)}(z)}{G_{0}(z)}+\frac{\hat{R}_{G_{0}}^{J \cap D_{0}-D(N)}(z)}{G_{0}(z)}\right) \\
& \leqq \limsup _{R \ni z \rightarrow-0} \frac{\hat{R}_{G_{z}}^{J \cap D_{0} \cap D(N)}(0)}{G_{z}(0)} \\
& \leqq \hat{R}_{1}^{J \cap D \cap D_{0}(N)}(0) .
\end{aligned}
$$

Therefore, by letting $N \rightarrow \infty$ and by (4), we have the equality (2).

2.2. Proof of Theorem 1. Suppose that $J$ is thin at $z=0$. Let $\pi$ be the projection from $W$ onto $D-\{0\}$. For every $\xi \in S$, we denote by $\xi$, the point in $W$ such that $\pi\left(\xi_{j}\right)=\xi$ and $\xi_{j} \in S_{j}(j=1, \cdots, p)$. Since the origin is a finely interior point of $S \cup\{0\}$, there exists the fine $\operatorname{limit} f-\lim _{S_{j} \ni \xi_{i \rightarrow 0}} G_{\xi_{j}}^{W}(\eta)$ for every $\eta \in W$ (cf. [2]), and hence the fine $\operatorname{limit} f-\lim _{S_{j \ni \xi j \rightarrow 0}} G_{\xi_{j}}^{W}(\eta)$ determines an element, 
denoted by $h_{j}(\eta)$, belonging to $\mathscr{Q}(W)$ for each $j=1, \cdots, p$. Thus, by the fact that $\operatorname{dim} \mathscr{Q}(W) \leqq p$, we have only to prove that the family $\left\{h_{1}, \cdots, h_{p}\right\}$ in $\mathscr{P}(W)$ is linearly independent. To see this, we define positive harmonic functions $h_{j k}$ on $S$ as follows: $h_{j k}(z)=h_{j}\left(z_{k}\right), \jmath, k=1, \cdots, p$, where $\pi^{-1}(z)=\left\{z_{1}, \cdots, z_{p}\right\}$ and $z_{k} \in S_{k}$. Then, we have only to prove the equality

$$
f-\lim _{S \ni z \rightarrow 0} \frac{h_{j k}(z)}{G_{0}(z)}=\delta_{j k}
$$

where $\delta_{j_{k}}$ is the Kronecker delta, since it instantly follows from (5) that the family $\left\{h_{1}, \cdots, h_{p}\right\}$ is linearly independent.

It is easily seen that

$$
G_{\xi}(z)=G_{z}(\xi)=\sum_{\jmath=1}^{p} G_{z_{k}}^{W}\left(\xi_{\jmath}\right)=\sum_{j=1}^{p} G_{\xi_{j}}^{W}\left(z_{k}\right)
$$

for each $z \in S$ and for each $k=1, \cdots, p$ (cf. [4]). Hence, by definitions of $h_{\text {, }}$ and $h_{j k}$, we obtain the equality

$$
G_{0}(z)=\sum_{j=1}^{p} h_{j k}(z)
$$

on $S$ for each $k=1, \cdots, p$. On the other hand, by (6), we have

$$
G_{\xi}(z) \geqq \sum_{j \neq k} G_{\xi_{j}}^{W}\left(z_{k}\right)
$$

for every $z \in S$ and for every $k=1, \cdots, p$. Hence, by (iv) and (v) of Proposition 1.1 and by maximum principle, we find that

$$
\hat{R}_{G_{\xi}}^{J}(z)=\hat{R}_{G_{\xi}}^{J \cup(0)}(z) \geqq \sum_{j \neq k} G_{\xi_{j}}^{W}\left(z_{k}\right) \quad(z \in S, k=1, \cdots, p),
$$

since $\sum_{j \neq k} G_{\xi_{j}}^{W}\left(z_{k}\right)$ is considered as a bounded harmonic function on $S$. Thus, by letting $\xi \rightarrow 0$ with respect to the fine topology and by (vi) of Proposition 1.1, we have

$$
\hat{R}_{G_{0}}^{J}(z) \geqq \sum_{j \neq k} h_{j k}(z) .
$$

Therefore, by virtue of Proposition 2.1, we obtain

$$
f-\lim _{S \ni z \rightarrow 0} \frac{\sum_{\jmath \neq k} h_{j k}(z)}{G_{0}(z)}=0 .
$$

It is easily seen that the equality (5) follows from (7) and (10). The proof is herewith complete.

\section{§3. Proof of Theorem 2}

3.1. We first give the following lemma which is useful in the sequel:

LEMMA 3.1. Let $F$ be an open Riemann surface, $\tilde{F}$ an unlimited covering 
surface of $F, E$ a subset of $F, s$ a positive superharmonic functin on $F$ and $\pi$ the canonical projection from $\tilde{F}$ onto $F$. Then, it holds that

on $\tilde{F}$.

$$
{ }^{F} \hat{R}_{s}^{E} \circ \pi={ }^{\tilde{F}} \hat{R}_{s \circ \pi}^{\pi-1}(E)
$$

Proof. Let $\tilde{u}$ be a positive superharmonic function on $\tilde{F}$ satisfying that $\tilde{u} \geqq s \circ \pi$ on $\pi^{-1}(E)$. Setting

$$
u(w)=\liminf _{x \rightarrow w} \inf \left\{\tilde{u}(z): z \in \pi^{-1}(x)\right\}
$$

on $F$, we find that $u$ is a positive superharmonic function on $F$ and $u \geqq s$ on $E$. Hence we have $\tilde{u} \geqq u \circ \pi \geqq{ }^{F} \hat{R}_{s}^{E} \circ \pi$ on $\tilde{F}$, which implies that

$$
\tilde{F} \hat{R}_{s \circ \pi}^{\pi-1}(E) \geqq{ }^{F} \hat{R}_{s}^{E} \circ \pi
$$

on $\tilde{F}$. Therefore, by a trivial relation ${ }^{F} \hat{R}_{s}^{E} \circ \pi \geqq{ }^{\tilde{F}} \hat{R}_{s \circ \pi}^{\pi-1(E)}$, we have the desired assertion.

3.2. Essential part of the proof of Theorem 2 is to prove the following proposition :

PROPOSITION 3.1. Suppose that $p=2$ and that $J$ is symmetric with respect to the real axis. If neither of $J \cap \boldsymbol{R}$ and $\boldsymbol{R}-J$ is thin at the origin, then $\operatorname{dim} \mathscr{P}(W)=1$.

Proof. Let $h$ be an element of $\mathscr{Q}(W)$ and $\pi$ the projection from $W$ onto $D-\{0\}$. For a point $z \in W$ which belongs to $S_{i}(i=1,2)$, we denote by $\bar{z}$ the point in $S_{\imath}$ whose projection coincides with $\overline{\pi(z)}$. Defining $\bar{h}$ by $\bar{h}(z)=h(\bar{z})$ on $W$, we find that $\bar{h} \in \mathscr{Q}(W)$.

First we show that $h(\in \mathscr{L}(W))$ is a constant multiple of $G_{0}(\pi(z))$ if $h=\bar{h}$. Let $\tau$ be the sheet interchange of $W$. Then, we find a positive constant $c$ such that

$$
c G_{0}(\pi(z))=h(z)+h \circ \tau(z)
$$

on $W$. Since $J \cap \boldsymbol{R}$ is not thin at the origin, by Lemma 3.1, (11) and (iii) of Proposition 1.1, we have

$$
\begin{aligned}
& c G_{0}(\pi(z))={ }^{D} \hat{R}_{c G_{0}}^{J \cap R}(\pi(z)) \\
& ={ }^{W} \hat{R}_{c G_{0}^{\circ}{ }^{\circ} \pi}^{\pi-1(J \cap R)}(z) \\
& \varliminf^{W} \hat{R}_{h}^{\pi-1(J \cap R)}(z)+{ }^{W} \hat{R}_{h}^{\pi}{ }^{-1} \tau \tau(J \cap R)(z) \\
& \leqq h(z)+h \circ \tau(z) \\
& =c G_{0}(\pi(z))
\end{aligned}
$$


on $W$ and, in particular,

$$
h(z)={ }^{W} \hat{R}_{h}^{\pi-1(J \cap R)}(z)
$$

on $W$. On the other hand, by (11), we also have

$$
h(z)=\frac{c}{2} G_{0}(\pi(z))
$$

for every $z \in \pi^{-1}(J \cap \boldsymbol{R})$, because $h=\bar{h}=h \circ \tau$ on $\pi^{-1}(J \cap \boldsymbol{R})$ except possibly a polar subset of $\pi^{-1}(J \cap \boldsymbol{R})$. By means of (12), (13), Lemma 3.1 and the assumption, we conclude that

$$
\begin{aligned}
h(z) & ={ }^{W} \hat{R}_{h}^{\pi^{-1}(J \cap R)}(z) \\
& ={ }^{W} \hat{R}_{(c / 2) G_{0}{ }^{-1}(J \cap \pi)}(z) \\
& \left.={ }^{D} \hat{R}_{(c / 2) G_{0}}^{J(\pi R}(z)\right) \\
& =\frac{c}{2} G_{0}(\pi(z))
\end{aligned}
$$

on $W$.

Next we consider the general case. Let $h \in \mathscr{P}(W)$ be a minimal function (cf. e.g. [2]). By the fact that $h+\bar{h}=\overline{h+\bar{h}}$ on $W$ and by the above observation, we find a positive constant $a$ such that

on $W$, and hence

$$
h(z)+\bar{h}(z)=a G_{0}(\pi(z))
$$

$$
h(z)=\frac{a}{2} G_{0}(\pi(z))
$$

on $\pi^{-1}(\boldsymbol{R}-J)$, because $\bar{z}=z$ on $\pi^{-1}(\boldsymbol{R}-J)$. Since $\boldsymbol{R}-J$ is not thin at the origin, by (15) and Lemma 3.1, we have

$$
\begin{aligned}
h(z) & \geqq{ }^{W} \hat{R}_{h}^{\pi-1(R-J)}(z) \\
& ={ }^{W} \hat{R}_{(a / 2) G_{0}{ }^{-1}(R-J)}^{\pi(z)}(z) \\
& ={ }^{D} \hat{R}_{(a / 2) G_{0}}^{(R-J) \cap}(\pi(z)) \\
& =\frac{a}{2} G_{0}(\pi(z))
\end{aligned}
$$

on $W$. Therefore, by the minimality of $h$, we find a positive constant $k$ such that

$$
h(z)=k G_{0}(\pi(z))
$$

on $W$, which implies that $\operatorname{dim} \mathscr{Q}(W)=1$.

3.3. Proof of Theorem 2. Take a minimal function $h$ in $\mathcal{P}\left(W_{p}\right)$, where $p=2^{m}(m \in N)$. Let $\theta$ be the covering transformation of $W_{p}$ : 


$$
\theta\left(w_{\imath}\right)=w_{\imath+1}, \quad(i \bmod p, i=1, \cdots, p)
$$

where $\pi^{-1}(w)=\left\{w_{1}, \cdots, w_{p}\right\}$ and $w_{i} \in S_{\imath}$ for $w \in D-\{0\}$. Set

$$
f_{\jmath}=\sum_{k=0}^{2 m-1-1} h \circ \theta^{2 k+\jmath} \quad(j=0,1),
$$

where $\theta^{0}=$ id.. Then we can consider $f_{0}$ as a function in $\mathscr{Q}\left(W_{2}\right)$. Hence, by Proposition 3.1, we find a positive constant $b$ such that

$$
f_{0}(z)=b G_{0}(\pi(z))
$$

on $W$, and hence, by the fact that $f_{1}=f_{0} \circ \theta$, we have

$$
f_{0}(z)=f_{1}(z)
$$

on $W$. Therefore, by the uniqueness of Martin's integral representation (cf. e.g. [2], [3], [5] etc), we can find an integer $l$ such that

$$
h=h \circ \theta^{2 l+1} \text {, }
$$

since $h \circ \theta^{2}$ is a minimal function for each $i=1, \cdots, p$. On the other hand, we can find two integers $\alpha$ and $\beta$ such that $\alpha(2 l+1)+\beta 2^{m}=1$. Therefore, by the fact that $\theta^{2^{m}}=\mathrm{id}$. and by (16), we have

$$
h=h \circ \theta \text {. }
$$

From this it follows that $\operatorname{dim} \mathscr{Q}\left(W_{p}\right)=1$.

3.4. By applying the same argument as in 3.3 and by the fact that $\operatorname{dim} \mathscr{P}\left(W_{n}\right) \leqq n$, we obtain the following :

THEOREM 3. Suppose that $p=2^{m} n$, where $m \in N$ and $n$ is an odd integer. Under the same condition for $J$ as in Theorem 2, it holds that $\operatorname{dim} \mathscr{P}\left(W_{p}\right) \leqq n$.

Remark. In Theorem 2, we can not omit the condition that $\boldsymbol{R}-J$ is not thin at $z=0$. For example, we assume that $p=2, J \subset \boldsymbol{R}$ and $\boldsymbol{R}-J$ is thin at $z=0$. Denote by $\left\{J_{n}^{\prime}\right\}_{n=1}^{\infty}$ the family of the connected components of $(\boldsymbol{R}-J) \cap$ $D-\{0\}$ and by $\bar{J}_{n}^{\prime}$ the closure of $J_{n}^{\prime}$ for each $n$. By replacing $\left\{J_{n}\right\}_{n=1}^{\infty}$ in 1.3 with $\left\{\bar{J}_{n}^{\prime}\right\}_{n=1}^{\infty}$, we construct a 2 -sheeted covering surface $W^{\prime}$ of $\{0<|z|<1\}$ in the same way as in 1.3. Then $\cup_{n=1}^{\infty} \bar{J}_{n}^{\prime}$ is thin at $z=0$, and hence Theorem 1 yields that $\operatorname{dim} \mathscr{Q}\left(W^{\prime}\right)=2$. Therefore we find that $\operatorname{dim} \mathscr{Q}(W)=2$ since $W$ is conformally equivalent to $W^{\prime}$.

\section{REFERENCES}

[1] J. Bliedtner and W. Hansen, Potential Theory, Springer-Verlag, 1986.

[2] M. BRELot, On Topologies and Boundaries in Potential Theory, Lecture Notes in Math., 175, Springer-Verlag 1971. 
[3] C. Constantinescu And A. Cornea, Ideale Ränder Riemannscher Flächen, Springer-Verlag, 1963.

[4] M. Heins, Riemann surfaces of infinite genus, Ann. of Math., 55 (1952), 296-317.

[5] L. Helms, Introduction to Potential Theory, Wiley-Interscience, 1969.

[6] S. Segawa, Harmonic dimension and extremal length, Kodai Math. J., 17 (1994), 256-261.

Department of Mathematics

FACULTY OF SCIENCE

KYOTO SANGYo University

Kamigamo-motoyama, Kitaku, Kyoto 603

JAPAN

Department of Mathematics

Daido Institute of Technology

Daido, Minami, Nagoya 457

JAPAN 
